\title{
Um estudo sobre os Fenômenos Afetivos e Cognitivos em Interfaces para Softwares Educativos
}

\author{
Magalí Teresinha Longhi \\ Eliseo Berni Reategui \\ Magda Bercht \\ Patricia Alejandra Behar

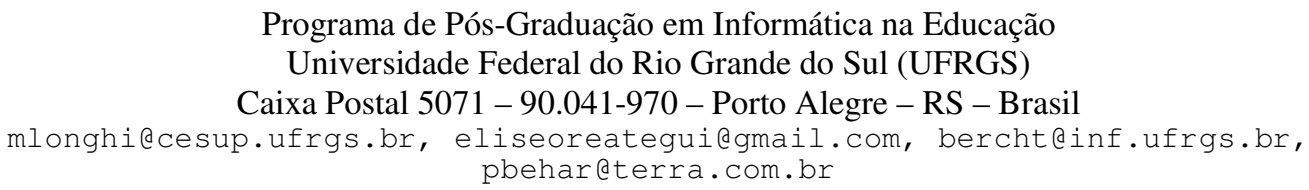

Resumo: Descobertas da Neurociência indicam que razão e emoção ou cognição e afetividade são elementos indissociáveis. São vários aspectos que devem ser considerados para sistematizar os conceitos de fenômenos afetivos, cada qual apoiado em teorias das mais diversas áreas do conhecimento. Este trabalho faz uma revisão histórica e teórica sobre a importância da afetividade na subjetividade humana, evidenciando sua influência na construção de conhecimento quando da utilização de interfaces em sistemas informáticos para a aprendizagem. Também apresenta o estadoda-arte da aplicação dos modelos afetivos em ambientes inteligentes de aprendizagem.

Palavras-chave: afetividade, fenômenos afetivos, cognição, aprendizagem, computação afetiva

\section{A study on Affective and Cognitive Phenomena in Educational Software Interfaces}

Abstract: New findings in Neuroscience indicate that reason and emotion, or cognition and affection, are not dissociable. Several aspects have to be considered to make systematic the concepts related to affective phenomena, each of them supported by theories in different knowledge areas. This paper reviews historic and theoretic work on the importance of affection in human subjectivity, making evident their influence in knowledge construction considering the use of interfaces in computational systems. It also presents the state of the art in the application of affective models in intelligent environments for learning.

Key-words: affectiveness, affective phenomena, cognition, learning, affective computing

\section{Introdução}

As tecnologias digitais de informação e comunicação vêm sendo apresentadas a educadores como o novo paradigma transformador nas formas de pensar e raciocinar e na construção de conhecimento. No entanto, como adverte Moraes (2002), estas tecnologias, ao prestigiarem tãosomente a função informativa e a instrucionista da educação, terminam por desconsiderar a função construtivista, isto é, os aspectos reflexivos e criativos.

Trata-se, então, de verificar como as Ciências Cognitivas podem se associar à Educação e à Informática de modo a desenvolver soluções computacionais de interação que permitam um ensinoaprendizagem mais efetivo.

A Informática, principalmente através da Inteligência Artificial em pesquisas que envolvem a representação do conhecimento, modelagem de processos lógicos, aprendizagem, percepção, comunicação e ação, apóia o desenvolvimento de teorias em que pensamento e aprendizagem são tomados como um conjunto de informações a serem processadas. Não se discute a importância 
destas pesquisas que, baseadas em fontes das Ciências Cognitivas, permitiram que a Educação viesse a estabelecer um novo paradigma de construção de conhecimento e novas práticas pedagógicas fossem desenvolvidas com auxilio do computador. Contudo, a maioria das aplicações construídas para esses propósitos dá mais ênfase aos processos cognitivos, não se importando ou desconsiderando os processos afetivos envolvidos.

As discussões sobre o papel da afetividade na subjetividade humana vêm se travando desde a Antiguidade, mas como elemento dissociável da cognição. Embora muitos pensadores, nas diversas épocas, insistissem nas relações existentes entre razão e emoção, apenas nas duas últimas décadas, importantes pesquisas em Neurociência, Psicologia e Ciências Cognitivas têm demonstrado como a afetividade está interligada de forma complexa com a cognição, constituindo papel fundamental em funções como tomada de decisão, memorização, e criatividade.

Ao discutir a relação entre cognição e afetividade na Psicologia, Pinto (2005) destaca que estas dimensões psicológicas funcionam "de maneira dinâmica e construtiva, sendo então de pouca importância dividi-las em fragmentos dissociados. Entende-se que, em cada experiência diária, o ser humano é cognitivo-afetivo ao mesmo tempo; estando em proporções variáveis, mais afetivo ou mais cognitivo, ou ambas somadas". Na perspectiva educacional, Arantes (2003) defende que a educação deve ocupar-se da construção e organização da dimensão afetiva dos aprendizes, já que "no trabalho educativo cotidiano não existe uma aprendizagem meramente

cognitiva ou racional", pois os aspectos afetivos não são deixados do lado de fora da sala de aula. Este trabalho procede a uma revisão histórica e teórica sobre a importância da afetividade na subjetividade humana, evidenciando sua influência na construção de conhecimento quando da utilização de interfaces em sistemas informáticos para a aprendizagem. $\mathrm{Na}$ próxima seção, é apresentada a visão dicotômica entre os aspectos afetivos e cognitivos. Já na Seção 3, são revistas as abordagens psicogenéticas que teorizam sobre a integração dos aspectos afetivos e cognitivos, enquanto que na seção seguinte é abordado como a Neurociência pode esclarecer esta integração. $\mathrm{Na}$ 5, são feitas considerações sobre a terminologia aplicada no estudo da afetividade e indicadas quais teorias são apropriadas para modelos computacionais. Na Seção 6, é relatado o estado-daarte do uso dos modelos afetivos em aplicações educacionais. Finalmente, na última Seção, são feitas algumas considerações sobre o assunto abordado.

\section{Visão Dicotômica da Afetividade e Cognição}

A discussão sobre o papel da afetividade na subjetividade humana começou com os estudos de Lao-Tzu, filósofo chinês do Século VI a.C., na civilização oriental, e com Sócrates, nos anos de 470 a 399 a.C., na ocidental (BERCHT, 2001). Platão (427-347 a.C.) fez uma primeira tentativa de estudo dos aspectos afetivos ao estruturar a alma: cognição, emoção e motivação (SCHERER, 1994). Mas, para esse filósofo, instintos, emoções e doenças constituíam obstáculos à racionalidade (NICOLA, 2005).

De um modo geral, da Grécia antiga até a modernidade, a razão está quase sempre dissociada da emoção. No século XVII, Descartes (1596-1650), considerado o pai da filosofia moderna, e Espinosa (1632-1677) revolucionaram o pensamento filosófico ao determinarem os processos mentais. Com seu célebre enunciado "penso, logo existo", Descartes evidenciava a perspectiva dualista entre corpo e mente. Por sua vez, Espinosa diria que "sentimentos são idéias e todo pensamento é ação; então qualquer ação é caracterizada pela idéia que o acompanha" (SCHERER, 1994).

Com o iluminismo do século XVIII, os pensadores passaram a refletir sobre as faculdades da mente humana. Leibniz (1646-1716) adotou o termo mônada para indicar a unidade de medida da força viva, espiritual e incorpórea que constituiria o fundamento da realidade; assim, cada mônoda era dotada de percepção, mas nem todas as percepções eram conscientes ao indivíduo. Locke (16321704) afirmava que todo conhecimento nasce da experiência do mundo externo e da reflexão interior. Considerava a mente, no momento do nascimento, como uma tábula rasa, isto é, uma folha em branco ainda a ser escrita. Hume (1711-1776) declarava que a moral era um sentimento, um 
estado afetivo não racional, que contribuía para a felicidade da sociedade. Rousseau (1712-1778) deu início à pedagogia moderna. Mas, para o filósofo, aprendia-se por si mesmo. O educador devia (1) evitar que o aluno entrasse em contato com influências perniciosas, (2) satisfazer a natural curiosidade do aluno e (3) predispor situações de vida de forma a favorecer seu crescimento. Kant (1724-1804), o mais importante filósofo da era moderna, afirmava que se "Deus tivesse feito o homem para ser feliz, não o teria dotado de razão”. O filósofo sugeria que a razão humana devia fazer uma autocrítica para estabelecer limites e determinar controles (NICOLA, 2005).

O início da pesquisa científica moderna sobre a natureza das emoções se deu com a publicação, em 1897, do livro Expressão das Emoções no Homem e nos Animais por Darwin (2004). Darwin acreditava que as expressões de emoções (faciais, por exemplo) são respostas emocionais observáveis em todos os membros de uma espécie que, associadas a outros comportamentos, determinariam a evolução da espécie. Entretanto, a primeira teoria físiológica da emoção foi proposta de forma independente por William James e Carl Lange em 1884. James e Lange argumentavam que o comportamento corporal desencadeado pelo evento emocional

(batimentos cardíacos, enrubescimento, etc.) produzia o sentimento da emoção, e não o contrário.

No começo do século XX, Walter Cannon e Philip Bard propuseram uma teoria alternativa à de James-Lange em que a experiência emocional e o comportamento corporal constituem processos paralelos sem relação causal direta (PINEL, 2005). Nessa época, os movimentos filosóficos impulsionaram os debates sobre pensamento, conhecimento, comportamento, razão e emoção. Mais tarde, com a consolidação de grandes teorias psicológicas - gestalt, psicanálise, behaviorismo, epistemologia genética, psicologia cultural e sócio-histórica -, a afetividade nas atividades cognitivas passa ser enfatizada.

Nas últimas décadas, dentre vários estudos, têm recebido destaque os desenvolvidos pelos psicólogos Howard Gardner (1994) e Daniel Goleman (1995). O primeiro pressupõe a idéia de "inteligências múltiplas" e o segundo a existência de uma "inteligência emocional". Entretanto, muitos pesquisadores não concordam com os pressupostos teóricos e epistemológicos por eles

apresentados (ARANTES, 2003).

\section{Integração da Afetividade e Cognição na Perspectiva Genética}

A psicogenética construtivista tem contribuído significativamente neste tema quando analisada nas perspectivas piagetiana, vygostskiana e walloniana. As abordagens genéticas passam a prestar atenção no papel da afetividade na cognição ao perceber que existe uma integração dos aspectos afetivos e cognitivos no funcionamento da psique humana.

Criador da Epistemologia Genética, Piaget (1896-1980) reconheceu que a afetividade é o agente motivador da atividade cognitiva. Para Piaget, a afetividade e a razão constituiriam termos complementares: "a afetividade seria a energia, o que move a ação, enquanto a razão seria o que possibilitaria ao sujeito identificar desejos, sentimentos variados, e obter êxito nas ações" (LA TAILLE, 1992; 2005).

Vygotsky (1896-1934) concebia a linguagem e a interação como elementos fundamentais da consciência e do aprendizado. O desenvolvimento pessoal seria operado em dois níveis: o do desenvolvimento real ou efetivo referente às conquistas realizadas e o do desenvolvimento potencial ou proximal relacionado às capacidades a serem construídas. Vygotsky é considerado, muitas vezes, cognitivista por ter se preocupado principalmente com os aspectos do funcionamento do pensamento. Entretanto, questionava o dualismo entre as dimensões afetivas e cognitivas quando menciona que a psicologia tradicional peca em separar os aspectos intelectuais dos afetivos-volitivos (OLIVEIRA, 1992). Vygotsky afirmava que "os processos pelos quais o afeto e o intelecto se desenvolvem estão inteiramente enraizados em suas inter-relações e influências mútuas" (OLIVEIRA, 1992, p.76). 
Wallon (1879-1962) trouxe contribuições significativas sobre o tema afetividade. A teoria de emoções proposta, com nítida inspiração darwinista, é simultaneamente social e biológica em sua natureza (DANTAS, 1992). Sua teoria envolve o estudo dialético, para determinar a natureza, assim como o estudo genético, para determinar as alterações funcionais. Afirmava que "a inteligência e a afetividade estão integradas: a evolução da afetividade depende das construções realizadas no plano da inteligência, assim como a evolução da inteligência depende de construções afetivas" (ARANTES, 2003). Para Wallon, a consciência afetiva dá origem à atividade cognitiva e que, durante o desenvolvimento humano, existem fases em que predominam o afeto e, em outras, a inteligência. Dantas (1992, p.86) resume o pensamento de Wallon com a seguinte afirmação: "A razão nasce da emoção e vive da sua morte".

\section{A Neuropsicologia estabelecendo relações entre Cognição e Afetividade}

A Neuropsicologia se desenvolveu pela convergência da Neurologia e da Psicologia com o objetivo de investigar como os sistemas cerebrais individuais se transformam em complexas atividades mentais. Nesta área, vários neurocientistas e psicólogos se associaram para tentar desvendar a relação existente entre funcionamento cerebral e atividades psicológicas como percepção, memória, linguagem, atenção, etc., levando-se em conta as variáveis biológicas, sociocultural e psico-emocional do ser humano.

Os neurocientistas Antônio Damásio e Joseph Le Doux demonstram e apresentam fortes evidências que os processos cognitivos e afetivos são indissociáveis. Le Doux sugere que os sentimentos e os pensamentos conscientes são parecidos e ambos são gerados por processos inconscientes e que a influência das emoções sobre a razão é maior que da razão sobre as emoções. Damásio (1996, 2000) faz três importantes considerações: (1) a emoção exerce influência nos processos mentais; (2) os sistemas cerebrais destinados à emoção estão intrinsecamente ligados aos sistemas destinados à razão; e (3) a mente não pode ser separada do corpo. A partir destas considerações, Damásio (1996) reescreve o postulado de Descarte: "Existo e sinto, logo penso".

Entre tantos outros pesquisadores das Ciências Cognitivas, Ortony, Clore e Collins (1988), Roseman (1990), Izard (1993), Sizer (2000), Scherer (2005) concordam que crenças e desejos exercem influências sobre os processos cognitivos que ativam as emoções.

\section{Terminologia e Modelos Computacionais para a Afetividade}

Um dos problemas no estudo da afetividade é a definição dos termos a ela relacionados: emoção, estados de humor, motivação, sentimento, paixão, atenção, personalidade, temperamento e outros tantos. O desafio é unir os conceitos utilizados nas diversas áreas de estudo dos fenômenos afetivos (psicologia, filosofia, sociologia, medicina, biologia, informática) de forma a homogeneizar e caracterizar as definições, podendo ser construídas categorias mais sólidas e formalizadas no consenso.

O número de definições científicas sobre o termo emoção é grande (até 1981 havia mais de cem definições listadas por Kleinginna e Kleinginna (apud Scherer, 2005)), já que os fenômenos afetivos são estudados em áreas de conhecimento diversas e não interdisciplinarmente. Scherer (2005) salienta que a dificuldade de diferenciar os fenômenos afetivos é similar ao problema de se entender as especificidades de uma linguagem em comparação a outras formas de comunicação. Por exemplo, Scherer trata da diferença entre emoção e estados de humor como sendo a primeira um episódio relativamente breve de resposta, sincronizada com a avaliação de um evento interno ou externo e, no segundo caso, como um estado afetivo difuso, de baixa intensidade, mas de longa duração, e sem causa aparente. Ortony et al. (1988) afirmam que emoção é uma reação com valoração a eventos, agentes ou objetos, cuja natureza particular é determinada pela maneira como a situação que a disparou é construída. Algumas emoções podem não possuir valoração (surpresa, interesse e aborrecimento). 
Alguns autores definem emoções descrevendo suas propriedades: "emoções podem ser caracterizadas por reações expressivas, como sorrisos, cenho franzido, dentes trincados, por reações fisiológicas, como aumento dos batimentos cardíacos, produção de lágrimas, calores e vermelhidão no rosto, por comportamentos instrumentais, como correr, buscar 'o conforto da mamãe', juntar as mãos, por comportamentos instrumentais situacionais, como digitar com força desmesurada uma tecla, gritar um impropério qualquer, por cognições, como pensamento de injustiça para si ou para outros, sensação de impotência frente a problemas, e por sentimentos que integram os fenômenos fisiológicos e cognitivos, como na tristeza (sensação de um aperto no peito, lágrimas nos olhos e a lembrança do evento que gerou tais sensações)" (BERCHT, 2001, p. 60)

Dessa forma, a afetividade é um termo utilizado para identificar um conjunto de fenômenos psíquicos que inclui as primeiras manifestações de tonalidades afetivas basicamente orgânicas (primeiras expressões de sofrimento e de prazer que a criança experimenta com a fome ou saciedade), bem como suas manifestações relacionadas ao social (sentimento, paixão, emoção, humor, motivação, etc) (ALMEIDA, 2002).

Na Informática, a área que se dedica aos estudos da afetividade e sua representação em computadores é conhecida por Computação Afetiva. Picard (1997) a define como a área que congrega as pesquisas da aplicabilidade da afetividade em sistemas não-biológicos, ou seja, o uso das emoções em diferentes aspectos nos sistemas computacionais desde o reconhecimento, representação e simulação até pesquisas que envolvem emoções nas interações homem-máquina.

Considerando-se o estágio atual da tecnologia digital, ainda é complexo tratar a afetividade em sistemas computacionais, e, em especial, os sistemas educacionais informatizados. Existe a necessidade da integração de estudos dos fenômenos associados nas Ciências Cognitivas, na Educação, e na Informática para determinar modelos que possam ser aplicados computacionalmente. A complexidade se dá principalmente porque cada indivíduo possui uma reação emocional e fisiológica diferente a cada situação. Influências cultural, social e genética também atuam na forma de como fenômenos afetivos são demonstrados. Além do mais, eles não ocorrem isoladamente. Dependendo da situação, os seres humanos têm reações geradas a partir de um conjunto deles.

Os modelos computacionais podem ser determinados considerando-se as quatro

principais correntes psicológicas (CORNELIUS, 2000) que estudam, explicam e definem, entre os fenômenos afetivos, as emoções: (1) perspectiva darwinista, discute as emoções como sendo universais e adaptativas; (2) perspectiva jamesiana, interpreta as emoções como sendo respostas às experiências corpóreas; (3) perspectiva social-construtivista, determina que as emoções são dependentes da cultura ou das regras de cada grupo social; e (4) perspectiva cognitivista, acredita que as emoções são baseadas na avaliação ou no significado cognitivo individual de um evento.

As teorias da abordagem cognitivista: Teoria de Roseman (1990) e Teoria OCC (ORTONY et al.,1988) são as mais usadas na implementação de emoções em computadores. As duas teorias são baseadas na avaliação dos eventos que antecedem as emoções, também conhecidas por Teoria do Appraisal.

A Teoria de Roseman (ROSEMAN, 1990) apresenta um modelo que distingue as emoções em Positivas e Negativas ao serem ocasionadas por eventos consistentes ou inconsistentes respectivamente. Então, se o objetivo for atingido, uma emoção positiva será produzida; caso contrário, uma emoção negativa. Um evento, cuja causa pode ser conhecida, desconhecida ou provável, pode acontecer por ter sido provocado pelo próprio sujeito; por outrem ou de forma circunstancial (não há controle sobre o evento). Eventos podem ocasionar desejos de recompensa ou punição e produzir um nível potencial para o tipo de emoção (forte ou fraca). O modelo de Roseman avalia os eventos somente de acordo com os objetivos e contempla o estado emocional Surpresa, não apresentado em outros modelos de appraisal. Possui uma estrutura simples, facilmente traduzida em regras que possam avaliar a causa do evento e, desse modo, determinar a emoção apropriada. Entretanto, o modelo apresenta problemas quando houver necessidade de avaliar mais de um evento para o mesmo sujeito. 
A teoria OCC (ORTONY et al.,1988) é largamente utilizada para sintetizar emoções e afetos conforme apresentado em (BERCHT, 2001, ADAMATTI, 2003, JAQUES, 2004). O modelo OCC (assim chamado por ter sido desenvolvido por Ortony, Clore e Collins) determina que uma emoção é derivada a partir da sua avaliação sob três aspectos: consequiência dos eventos, ação dos agentes e aparência dos objetos. As percepções emocionais de um agente são valoradas a partir de seus objetivos, padrões e preferências. O cálculo da intensidade da emoção é derivado a partir de variáveis globais (senso de realidade, proximidade, etc.) e locais (probabilidade do evento ocorrer, esforço para atingir o objetivo, possibilidade da realização do objetivo, etc.). Além dos objetivos, o modelo OCC avalia as preferências e os padrões como gostar/não gostar, aprovar/desaprovar, prazer/desprazer. É mais simples e completo no seu entendimento em relação ao modelo de Roseman.

\section{Aplicação dos Modelos Afetivos nas Interfaces de Softwares Educativos}

A área da Computação Afetiva busca reconhecer e representar a afetividade na interação homem-máquina-homem e também investiga como expressar a afetividade. Os fenômenos afetivos podem ser inferidos através de dispositivos de entrada de dados (por exemplo, câmaras que analisam as expressões faciais), analisados através da aplicação de modelos afetivos e, então, gerados estímulos através de sinais que são interpretados pelos seres humanos como respostas adequadas. Picard (1997) aponta ainda a possibilidade da síntese (ou simulação) dos estados afetivos, isto é, a máquina deve saber detectar eventos que disparam um estado afetivo, entender porque está suportando determinado estado afetivo, agir ao estado afetivo reconhecido e aprender com o estado afetivo vivenciado.

O uso de tecnologia para reconhecer afetividade com mais acuidade, ou seja, perceber, identificar e medir fenômenos afetivos, ainda carece de muito estudo e do desenvolvimento de sensores e interfaces que associados às técnicas de reconhecimento de padrões, processamento de sinais (som e imagens) e algoritmos computacionais poderão avaliar e responder os estados afetivos dos aprendizes em tempo real (PICARD et all, 2004). Sendo assim, a inferência computacional de fenômenos afetivos, principalmente em ambientes educacionais, é um dos desafios da Computação Afetiva. Há necessidade de uso e desenvolvimento de novas tecnologias que ajudem a perceber, identificar, medir, expressar, simular a afetividade em situações de aprendizagem.

A maioria dos sistemas educativos trata do fenômeno afetivo classificado como emoções. Do conjunto de emoções, apenas as conhecidas como básicas (1) (a saber: raiva, medo, alegria, tristeza, nojo, vergonha e culpa) são aplicadas. Essas podem ser inferidas por vários mecanismos como gestos e expressões faciais, linguagem (textual, oral e de sinais), tensão muscular, condutividade da pele, respiração, ritmo cardíaco, temperatura, movimentos oculares, e pelo comportamento observável (PICARD et al., 2004). O uso de equipamentos com sensores visuais (video-câmeras), de áudio (microfones) e fisiológicos (cadeiras sensíveis à pressão do corpo, luvas que captam a condutividade da pele, mouse sensível à "qualidade" de pressão, EEG, ECG, ERP, termógrafos, aparelhos para verificar pulsação, respiração, dilatação da pupila) ainda são onerosos e de difícil utilização, além de desconfortáveis e poderem interferir no reconhecimento das emoções.

As pesquisas empreendidas na Computação Afetiva voltadas para a Informática na Educação referem-se principalmente na construção de agentes afetivos pedagógicos, com recursos ou não de animação e multimídia, antropomórficos ou não, sendo que, na maior parte deles, em ambientes inteligentes de aprendizagem (2). Jaques (2004) apresenta PAT, que age no sistema educacional MACES, como um agente afetivo personificado numa figura feminina, inferindo as emoções do aluno por seu comportamento observável (tempo de execução de uma atividade, sucesso ou falha na execução de um exercício e pedido de ajuda), isto é, pelas ações do aluno na interface do sistema.

Os agentes pedagógicos afetivos inseridos neles podem manter um diálogo verbal e nãoverbal, demonstrar uma ação enquanto "fala", contemplar, afirmar/negar com a cabeça, mostrar algumas expressões faciais, características estas que representam um retorno às interações do aluno, uma vez que caracterizam componentes naturais do diálogo humano. Vicent (PAIVA e MACHADO, 
1998), Adele (JOHNSON et al., 1998), Steve (RICKEL e JOHNSON, 1998), Cosmo (LESTER et al., 1997), Herman the Bug (LESTER e STONE, 1997), PAT (JAQUES, 2004), WHYFLY (DOSWELL, 2004) são exemplos de agentes pedagógicos afetivos representados por personagens animados antropomórficos, ou não.

As emoções, na maioria dos ambientes inteligentes de aprendizagem, são reconhecidas através do comportamento observável. A análise é feita aplicando-se modelos computacionais baseados na teoria do appraisal, mais precisamente no modelo OCC (ORTONY et al., 1988). Vários frameworks ou estruturas básicas de sistemas computacionais que comportam alguns fenômenos afetivos têm sido desenvolvidos na última década e apresentados a seguir.

O framework EM proposto por Reilly (1996) é composto por um sistema gerador de emoções (24 tipos) em que as variáveis de entrada (percepções do ambiente, objetivos, padrões, atitudes, memória sensorial, postura corporal, relacionamentos, modelos dos outros agentes) determinam quais emoções são positivas (alegria, esperança, felicidade, prazer, amor, satisfação, alívio, orgulho, admiração, gratidão e agradecimento) e quais negativas (aflição, receio, medo, pena, ressentimento, desapontamento, vergonha, reprovação, remorso, irritação, ódio, frustração e assustado).

Velásquez (1997) propôs o modelo Cathexis baseado em redes comportamentais que incorpora sistemas proto-especialistas (3) para representar emoções, temperamentos e estados de humor. Cathexis diferencia emoções básicas (fúria, medo, tristeza, alegria, nojo e surpresa) de emoções mistas ou secundárias determinadas quando ocorrer mais de uma emoção protoespecialista ao mesmo tempo, sem que haja dominância de uma delas (por exemplo, arrependimento pode ser uma variedade de tristeza mesclada com medo e às vezes fúria).

O modelo sócio-psicológico proposto por Rousseau e Hayes-Roth (1998) define os traços de personalidade, estados de humor e atitudes. Os traços de personalidade correspondem aos padrões de comportamento do indivíduo no ambiente computacional. O modelo suporta emoções e estados de humor que são valorados positiva ou negativamente dependendo do prazer ou desprazer que o indivíduo vivencia no ambiente.

El-Nasr et al. (2000) apresentam o modelo FLAME composto pelos módulos emocional, de aprendizado e de tomada de decisão. As percepções do agente sobre os eventos externos são avaliadas pelo módulo emocional e de aprendizado. $\mathrm{O}$ modelo faz uma combinação entre as teorias de Roseman (ROSEMAN, 1990) e OCC (ORTONY et al., 1988).

Kshirsagar e Magnenat-Thalmann (2002) construíram o PEM (P-personalidade, Eemocional, M-estados de humor) em agentes conversacionais tridimensionais. Para determinar a personalidade, o modelo PEM inclui o modelo FFM (Five Factor Model); as emoções, o modelo

OCC; e os estados de humor na valoração: positivo, negativo e neutro.

O framework EMA de Gratch e Marselha (2004) é baseado na teoria OCC (ORTONY et al., 1988) para determinar instâncias emocionais como: esperança, alegria, medo, aflição, irritação e culpa.

Apesar dos estudos envolvendo a incorporação de emoções e estados de humor em agentes afetivos pedagógicos (ou não pedagógicos), vários desafios se apresentam no reconhecimento e expressão da afetividade na construção de interfaces homem-máquina. A complexidade da construção de sistemas computacionais que reconhecem e geram fenômenos afetivos se dá principalmente porque cada indivíduo possui uma reação emocional e fisiológica diferente a cada situação. Sem dúvida, o reconhecimento e expressão da afetividade são elementos essenciais na comunicação e está estritamente relacionado a temas como: estar ou não motivado, estar ou não aborrecido, estar ou não indiferente, estar ou não frustrado, fatores importantes a serem considerados na aprendizagem favorecendo a intervenção dos professores,

quando necessário. 


\section{Algumas Considerações}

Descobertas da neurociência indicam que razão e emoção são elementos indissociáveis. Uma redução ou excesso de afetividade resulta em problemas na cognição. Também não é verdade que a razão se sobreponha à emoção. Pelo contrário, a emoção pode auxiliar no raciocínio principalmente em questões pessoais e sociais que envolvam conflitos, revelando sua importância na tomada de decisões (DAMÁSIO, 2000) e, por sua vez, nos processos de aprendizagem.

Empenho das diversas áreas para tentar explicar a afetividade, considerando-se as diferenças pessoais, culturais e de linguagem, acabou produzindo conceitos isolados. Torna-se importante criar um mínimo de consenso sobre a definição dos diferentes tipos de fenômenos afetivos. Mesmo assim, modelos computacionais para tratamento dos fenômenos afetivos, mais precisamente das emoções e dos estados de humor, estão sendo desenvolvidos e empregados em ambientes inteligentes de aprendizagem direcionados ao domínio de conhecimento a ser aplicado.

Entretanto, pouca importância é dada ao estudo da afetividade nas interações realizadas através de ambientes virtuais de aprendizagem(4), os quais favorecem a construção de conhecimento e permitem o desenvolvimento de práticas pedagógicas mediadas por tecnologias digitais através da interação, cooperação e colaboração dos sujeitos participantes. Tampouco, verifica-se a preocupação de introduzir os estudos de afetividade no desenvolvimento de interfaces para a Internet. Recursos de imagens, sons e cores são os mais utilizados para provocar e expressar afetividade.

Picard (2003) prevê que, por muito tempo ainda, os projetistas continuarão a ignorar a afetividade no desenvolvimento de aplicativos computacionais. No entanto, aqueles que se arriscarem a construir interfaces que reconheçam e expressem fenômenos afetivos como se fossem inatas a elas, contribuirão com o futuro de interfaces inteligentes, importantes na tomada de decisão e de uma aprendizagem mais efetiva.

\section{Referências Bibliográficas}

ADAMATTI, D. F. AFRODITE: Ambiente de Simulação Baseado em Agentes com Emoções. Instituto de Informática. Universidade Federal do Rio Grande do Sul. Porto Alegre, RS. Fev, 2003. Dissertação de Mestrado.

ALMEIDA, A. M. S. O que é afetividade? Reflexões para um conceito. In: Educação On-line. Fonte ANPED. (Incluído em 24/2/2002 e obtido em 4/5/2007 no endereço http://www.educacaoonline.pro.br/o_que_e_afetividade.asp). 2002

ARANTES, V. A. Afetividade e Cognição: Rompendo a Dicotomia na educação; In VIDETUR, n. 23. Porto/Portugal, Mandruvá, 2003, (obtido em 4/5/2007 no endereço http://www.hottopos.com/videtur23/valeria.htm).

BEHAR, P. A. ; WALQUIL, M.; BERNARDI, M; MORESCO, S. Refletindo sobre uma metodologia de pesquisa para AVA's.In: Congresso Internacional de Qualidade de Educação a Distância, 2005, São Leopoldo. Anais. São Leopoldo: Unisinos, v. 1. 2005.

BERCHT, M.. Em Direção a Agentes Pedagógicos com Dimensões Afetivas. Instituto de Informática. UFRGS. Tese de Doutorado. Dezembro, 2001.

BURLESON, W., PICARD, R.W., PERLIN, K., LIPPINCOTT, J., A Platform for Affective Agent Research. In: Workshop on Empathetic Agents, International Conference on Autonomous Agents and Multiagent Systems, Columbia University, New York. July, 2004

CORNELIUS, R. R. Theoretical approaches to emotion. In: Proceedings of the ISCA Workshop on Speech and Emotion, Belfast. 2000.

DANTAS, H. A Afetividade e a Construção do Sujeito na Psicogenética de Wallon. In: LA TAILLE, Y. (Org.) Piaget, Vygotsky, Wallon: teorias psicogenéticas em discussão. São Paulo: Summus, 1992. p. 75-98.

DAMÁSIO, A. O Erro de Descartes: emoção, razão e o cérebro humano. Tradução: Dora Vicente e Georgina Segurado. São Paulo: Cia das Letras, 1996. 
DAMÁSIO, A. O Mistério da Consciência: do corpo e das emoções ao conhecimento de si. Tradução: Laura Teixeira Motta. São Paulo: Cia das Letras, 2000.

DARWIN, C. A Epressão das Emoções no Homem e nos Animais. São Paulo: Cia das Letras. 2004.

DOSWELL, J. Pedagogical Embodied Conversational Agent. In: ICALT'04 - Proceedings of the IEEE Internetional Conference on Advanced Learning Technologies. p. 774-776. Joensuu, Finlândia. 2004.

EKMAN, P.. Basic Emotions In: T. Dalgleish and T. Power (Eds.) The Handbook of Cognition and Emotion. p. 45-60. Sussex, U.K.: John Wiley \& Sons, Ltd. 1999.

EL-NASR, M. S.; YEN, J.; IOERGER, T. R. FLAME-fuzzy logic adaptive model of emotions. Autonomous Agents and Multi-Agent Systems, 3(3):219-257. 2000.

GARDNER, H. Estruturas da Mente: a teoria das inteligências múltiplas. Trad. Sandra Costa. Porto Alegre: Artes Médicas Sul, 1994.

GOLEMAN, D. Inteligência Emocional. 46. ed. Rio de Janeiro: Objetiva, 1995.

IZARD, C.E.. Basic emotions, relations among emotions, and emotion-cognition relations. In: Psychology Review. 99:561-65. 1992.

JAQUES, P. Using an Animated Pedagogical Agent to Interact Affectively with the Student. Tese de doutorado. Porto Alegre: PPGC da UFRGS, 2004.

JOHNSON, L. et al. Pedagogical Agents on The Web. In: ITS'98 Workshop on Pedagogical agents, 4,1998. San Antônio. Anais. San Antonio: p.2-7. 1998.

KSHIRSAGAR, S.; MAGNENAT-THALMANN, N. A Multilayer Personality Model. In Symposium on Smart Graphics: 107-115. Hawthorne, NY, USA. 2002.

LA TAILLE, Y. Desenvolvimento do juízo moral e a afetividade na teoria de Jean Piaget. In: LA TAILLE, Y. (Org.) Piaget, Vygotsky, Wallon: teorias psicogenéticas em discussão. São Paulo: Summus, 1992. p. 47-73.

LA TAILLE, Y. Desenvolvimento do juízo moral. In: Coleção Memória da Pedagogia: Jean Piaget. Ed. Ediouro: Rio de Janeiro; Ed. Segmento-Dueto: São Paulo; 1 (1), 2005. p.76-88.

LE DOUX, J. Emotion: Clues from the Brain. In: Annual Review Psychology. 46:209-235, 1995. LESTER, J.; STONE, B. Increasing Believability in Animated Pedagogical Agents. In: International Conference on Autonomous Agents, 1. 1997. Proceedings. Marina del Rey: [s. n.], 1997. p. 16-21.

LESTER, J. et al. Cosmo: A Life-like Animated Pedagogical Agent with Deictic Believability. In: IJCAI; Workshop on Animated Pedagogical Agents: Making them Intelligent, 1997, Nagoya. Proceedings... San Francisco: Morgan Kaufmann, p. 61-69. 1997.

MINSKY, M. The society of mind. New York: Simon \& Schuster Paperbacks. 1988.

MORAES, M. C. Tecendo a rede, mas com que paradigma? In: MORAES, Maria Cândida (Org.). Educação a distância: fundamentos e práticas. Campinas: Nied/Unicamp, 2002.

NICOLA, U. Antologia Ilustrada de Filosofia: das origens à idade moderna. Ed. Globo. São Paulo, 2005.

OLIVEIRA, M. K.. O problema da afetividade en Vygostsky. In: LA TAILLE, Y. (Org.) Piaget, Vygotsky, Wallon: teorias psicogenéticas em discussão. São Paulo: Summus, 1992. p. 75-84.

PAIVA, A.; MACHADO, I. Vincent, an autonomous pedagogical agent for on-the-job training. In: Conference on Intelligent Tutoring Systems, 4., 1998, San Antonio. Proceedings...Berlin:

Springer-Verlag, 1998.

PICARD, R. W. Affective Computing. Cambridge: MIT Press. 1997.

PICARD, R.W. Affective Computing: challenges. In: International Journal of Human-Computer

Studies, Volume 59, Issues 1-2, July 2003, pp. 55-64. 2003.

PICARD, R.W.; PAPERT, S.; BENDER, W.; BLUMBERG, B.; BREAZEAL, C.; CAVALLO, D.; MACHOVER, T.; RESNICK, M.; ROY, D.; STROHECKER, C. Affective Learning-A

Manifesto. BT Technical Journal, Volume 22, No. 4, pp. 253-269. October, 2004.

PINEL, J.P.J. Biopsicologia. 5. ed. Porto Alegre: ArtMed., 2005.

PINTO, F.E.M. Os (des) afetos da inteligência... O possível diálogo entre cognição e afetividade. Publicação UEPG Ciências Humanas, Ciências Sociais Aplicadas, Linguagem, Letras e Artes, Ponta Grossa, 13 (1) 7-12, jun. 2005.

RICKEL, J.; JOHNSON, L. Steve: A Pedagogical Agent for Virtual Reality. In: International Conference on Autonomous Agents, 2. 1998, Minneapolis. Proceedings... New York: ACM Press, p. 165-172. 1998. 
REILLY, N. Believable Social and Emotional Agents. Ph.D. Thesis. Technical Report CMU-CS96-138, School of Computer Science, Carnegie Mellon University, Pittsburgh, PA. 1996. ROUSSEAU, D.; HAYES-ROTH, B.. A social-psychological model for synthetic actors. In: Autonomous Agents 1998, Minneapolis, MN. (p 165-172).1998.

SCHERER, K.R. Plato's legacy: Relationships between cognition, emotion and motivation. Geneva Studies in Emotion and Communication, 9(1). 1-7. 1995.

SCHERER, K. What are emotions? And how can they be measured? In: Social Science Information 44 (4), 695-729. 2005.

SIZER, L. Towards a computational theory of mood. In: British Journal of Philosophy of Science. 51. 743-769. 2000.

VELÁSQUEZ, J. D. Modeling emotions and other motivations in synthetic agents. In Proceedings of the 14th National Conference on Artificial Intelligence and 9th Innovative Applications of Artificial Intelligence Conference (AAAI-97/IAAI-97). 1997.

\section{Notas de Texto:}

(1) Scherer (2005) descreve o fenômeno afetivo como sendo composto pelos tipos: Preferências, Atitudes ou Posturas, Estados de humor, Disposições Afetivas, Posturas Interpessoais, Emoções Estéticas e Emoções Utilitárias. O conjunto de emoções utilitárias de Scherer coincide com o conjunto de emoções básicas propostas por Ekman (1999).

(2) Os ambientes inteligentes de aprendizagem são baseados nos Sistemas Tutores Inteligentes desenvolvidos para um determinado conteúdo educacional e baseados nos modelos de conteúdo instrucional e estratégias de ensino de acordo com o modelo de aluno.

(3) Termo introduzindo por Minsky (1988).

(4) Behar et al. (2005) consideram o Ambiente Virtual de Aprendizagem (AVA) como sendo constituído por uma infra-estrutura tecnológica (interface gráfica, comunicação síncrona/assíncrona e outras funcionalidades) e por todas as relações (afetivas, cognitivas, simbólicas, entre outras) estabelecidas pelos sujeitos participantes, tendo como foco principal a aprendizagem. 